University of New Hampshire

University of New Hampshire Scholars' Repository

$10-1991$

\title{
Sidescan Sonar Image Enchancement Using a Decomposition Based on Orthogonal Functions. Applications with Chebyshev Polynomials
}

\author{
Pierre Cervenka \\ University of California - San Diego \\ Christian de Moustier \\ University of California - San Diego
}

Follow this and additional works at: https://scholars.unh.edu/ccom

Part of the Computer Sciences Commons, and the Oceanography and Atmospheric Sciences and Meteorology Commons

\section{Recommended Citation}

Cervenka, Pierre and de Moustier, Christian, "Sidescan Sonar Image Enchancement Using a Decomposition Based on Orthogonal Functions. Applications with Chebyshev Polynomials" (1991). IEEE Oceans. 170.

https://scholars.unh.edu/ccom/170

This Conference Proceeding is brought to you for free and open access by the Center for Coastal and Ocean Mapping at University of New Hampshire Scholars' Repository. It has been accepted for inclusion in Center for Coastal and Ocean Mapping by an authorized administrator of University of New Hampshire Scholars' Repository. For more information, please contact Scholarly.Communication@unh.edu. 


\title{
SIDESCAN SONAR IMAGE ENCHANCEMENT USING A DECOMPOSITION BASED ON ORTHOGONAL FUNCTIONS. APPLICATIONS WITH CHEBYSHEV POLYNOMIALS
}

\author{
P. Cervenka and C. de Moustier \\ Marine Physical Laboratory \\ Scripps Institution of Oceanography \\ University of California, San Diego \\ 9500 Gilman Drive \\ La Jolla, California 92093-0205 U.S.A.
}

Abstract: A method is presented to remove from sidescan sonar images of the seafloor, artifacts that are clearly unrelated to the backscattering properties of the seafloor. A spectral analysis performed on a ping by ping basis proved to be well suited to the problem. The technique relies on a decomposition using Chebyshev polynomials. This stochastic method does not require a priori knowledge of deterministic parameters. It deals with the low spatial frequency components of the image whose wavelengths are not very small compared to the swath width. Applications to sidescan sonar images obtained with the SeaMARC II system are presented.

\section{INTRODUCTION}

Sidescan sonar images of the seafloor typically consist of a series of lines, one per transmission-reception cycle, displayed perpendicular to the survey track. On each side of the track, a single line segment represents the echoes received from the seafloor for a given ping as a function of slant range (time), or horizontal range if the appropriate corrections have been applied. In addition, a time varying gain is typically used to compensate for transmission losses due to spherical spreading and absorption of sound waves in water. However, if no other corrections are made, the resulting images usually suffer from numerous distortions and artifacts because: the survey track is rarely straight and the attitude of the tow fish (roll, pitch, yaw) changes with time; the beam patterns of the sonar are not uniform in the angular sector of interest, in addition their side or back lobes may contribute to cross-talk between the two sides or may pick up echoes reflected from the sea surface; and various forms of noise or external interferences are added to the process. Several image processing techniques have been used to correct or alleviate these artifacts (e.g. [1-7]).

In this paper we are first concerned with artifacts appearing as abrupt amplitude changes between adjacent pings, giving the impression that the grey level of these pings has a constant offset with respect to the neighboring pings. Actually, such artifacts are most likely due to the yaw, and to a lesser extent the pitch, of the tow fish: as the bearing of the sonar changes between transmission and reception, the high azimuthal directivity of the arrays (typically $=<2^{\circ}$ ) induces drastic variations in the level of the received signal (Fig. 1). These attitude fluctuations occur while the stream of echoes from the seafloor is being recorded, so that the perturbation is more complicated than a simple offset as mentioned above. However, the yaw and pitch periods are not very small compared to the ping cycle so that the size of the resulting multiplicative patterns is not very small compared to the swath width.

We also address artifacts that are nearly invariant in the along-track direction. Incorrect time varying gain corrections, as well as poor or inexistent beam pattern corrections lead to acrosstrack variations of signal intensity that remain coherent along-track over whole traverses. In some instances, the angular dependence of seafloor backscatter produces a similar effect. As in the case of yaw, the resulting across-track patterns are likely to be slowly varying on distances commensurate with the swath width. The correction method presented below is based on this assumption.

A direct approach to removing these artifacts is the causal method whereby deterministic corrections are derived from available parameters such as the recorded position of the sonar in the water column, the estimated propagation losses or the calibrated directivity of the transducers. However, in most cases these causal phenomenons are inaccurately quantized and residual artifacts are still visible in the corrected image.

Our approach is purely stochastic and does not require a priori knowledge of the fish's attitude or position in the water column. It deals with the low spatial frequency components of the image whose wavelengths are not small compared to the half width of the swath. This method does not address purely geometric distortions, cross-talk or interferences from other sound sources. It can be implemented directly or at a post-processing stage, i.e. after deterministic corrections have been applied.

In this paper we show how a Chebyshev polynomial decomposition applies to this problem by spectral analysis of the spatial characteristics of the image (Section II). The processing algorithm is described in section III, and an example of its applications to sidescan sonar data recorded with the SeaMARC II system, operated by the Hawaii Institute of Geophysics, is given in Section IV.

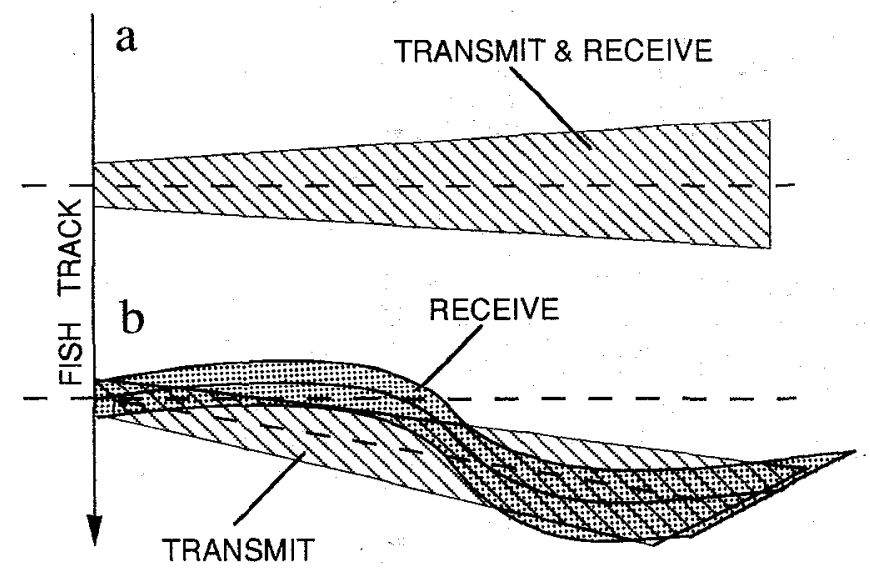

Figure 1 - Effect of fish attitude on acousctic backscatter geometry. A one-sided insonification pattern is shown for: (a) the ideal case in which transmit and receive beam patterns overlap perfectly and are perpendicular to the track; (b) the transmit beam is not perpendicular to the track and the receive pattern shows the effect of yaw and pitch motions. 


\section{SPECTRAL ANALYSIS}

In the following analysis, we consider that the port and starboard sides are independent and the corresponding images are treated as if coming from two one-sided systems. This approximation is valid if one excludes cross-talk effects which are not addressed here. For each side, the image is cropped to a rectangular frame which is filled without data gaps along or across track, so that continuous records can be processed in both perpendicular directions. Images are displayed in terms of horizontal range derived with the classical flat bottom assumption, although the across-track expansion could have been done just as well in terms of slant range: the pertinent fact is that data are framed in a rectangle and fully fill it. In addition, it is assumed that each of these individual rectangles covers an area of the seafloor whose acoustical properties remain relatively homogeneous. In other words, any portion taken within the whole rectangle, whose characteristic size is on the order of half a swath width, features the same type of bottom. This requires, for example, that the relief fluctuations are at least an order of magnitude smaller than the local water depth.

Because sidescan sonars gather information on a ping by ping basis, the backscatter images are made of contiguous across-track line segments, with each segment constituting a quasi continuous element of information. Consequently, to correct the erratic signal amplitude variations between adjacent pings we make a 1-D spectral analysis of each line (ping); then the same order components are processed in the perpendicular direction (along-track).

The yaw of the fish introduces a multiplicative "noise" which adds low spatial frequency components in the across-track signal spectra. Hence, the low frequency content of the recorded signal is partly due to the very nature of the seafloor and partly due to the artifacts introduced by the sonar's motion. However, unless the sea bottom is very anisotropic, the fast along-track evolution of these components is mostly the signature of the artifacts. Thus, an along-track low-pass filtering of the very low spatial frequency components should not remove any seafloor information, but correct the artificial short range fluctuations.

The same spectral analysis is also performed on a single record made of cumulated pings in the along-track direction. We assume that the seafloor does not feature an anisotropy that would lead to significant along-track averages of the low frequency coefficients. Accordingly, the residual content is attributed to artifacts that are removed over the entire frame. However, before performing this operation, the averaged coefficients are tapered to reduce the impact of the filter on the "higher" frequencies. This precaution must be taken to ensure that artificial features are not introduced. For example, as explained in Section IV, surface reflected echoes can appear as a narrow line running along-track. If this line undergoes small lateral fluctuations, a non tapered correction would introduce ghost images of this line over the whole frame. "Natural" narrow features could also introduce such artifacts. Avoiding this drawback is the main advantage of the spectral analysis compared to the direct approach: a conventional method consists of stacking the backscatter amplitudes along track and, assuming the resulting across-track distribution is statistically uniform, performing a suitable multiplicative correction over the whole frame. Our method is basically similar, but it limits the uniformity assumption to the weighted low spatial frequency components.

With these general guidelines, one can think of decomposing the framed image into a set of orthogonal basis functions by relating the concept of "low" or "high" spatial frequencies to the intuitive notion implied by the number of median value crossings, or the number of sign changes of the second derivative, which characterize the corresponding basis function over a range of abscissa commensurate with the swath width. We now proceed with the choice of a decomposition basis.

Let us denote $s_{y}(x)$ the $y^{\text {th }}$ record, defined over the interval $\left[x_{\min }, x_{\max }\right]$ (constant width $=$ rectangular image). We want to approximate these records by a function $\sigma_{y}(x)$ which is a linear combination of a set of $\mathrm{N}$ functions $F_{j}(x)$ ordered in the sense of the spatial frequencies mentioned previously:

$$
\sigma_{y}(x)=\sum_{j=0}^{N-1} a_{j}[y] F_{j}(x)
$$

This approximation is performed through a classical least squares fitting process, whereby minimizing the expression

$$
\int_{x_{\min }}^{x_{\max }}\left[s_{y}(x)-\sigma_{y}(x)\right]^{2} d x,
$$

amounts to finding the $\mathrm{N}$ coefficients $a_{j}$ by solving the corresponding set of normal equations:

$$
\sum_{j=0}^{N-1} a_{j}[y] S_{j, k}=G_{k}[y], \quad k=0,1, \ldots, N-1
$$

with

$$
S_{j, k}=\int_{x_{\min }}^{x_{\max }} F_{j}(x) F_{k}(x) d x, \quad G_{k}[y]=\int_{x_{\min }}^{x_{\max }} s_{y}(x) F_{k}(x) d x .
$$

However, this system is generally difficult to solve because ill-conditioned. On the other hand, we need a decomposition $\left\{a_{j=0, N-1}\right\}$ such that adding or removing members of the highest order's in the family of basis functions does not change the coefficients computed at the lower orders. This essential property is unique to a decomposition based on orthogonal functions. Then, filtering operations can make sense as the values of the components $a_{j}$ do not depend on the total number, $N$, of functions kept to perform the decomposition. Moreover, each of these spectral values can be obtained independently and easily by computing the corresponding Fourier coefficient.

By definition, a set of functions $F_{j}(x)$ is said to be orthogonal with respect to the weight factor $w(x)>0$ and over the interval [ $\left.x_{\min }, x_{\max }\right]$ if the integrals $S_{j, k}$, modified by including $w(x)$ in the integrands, are all null whenever $j \neq \neq$. Therefore, a system similar to Eq.(3) can be written. It is now diagonal and straightforward to solve. Except for a multiplicative constant, the unknown coefficients $a_{j}$ are the Fourier coefficients which identify with the integrals $G_{j}$ (Eq.4) modified by the weight factor $w$ :

$$
a_{j}[y] \equiv \int_{x_{\min }}^{x_{\max }} w(x) s_{y}(x) F_{j}(x) d x
$$

Within the family of orthogonal functions, we chose the Chebyshev polynomials $T_{n}(X)$ which are very well suited to approximate bounded functions. Chebyshev polynomials are orthogonal in the interval $[-1,1]$ over a weight $\left(1-X^{2}\right)^{-1 / 2}$. This weight factor gives the necessary emphasis on both ends of the bounded function that we want to approximate, so that there is no "leakage" of accuracy at the edges. The first members of this family are:

$$
\begin{aligned}
& T_{0}=1 ; \\
& T_{1}=X ; \\
& T_{2}=2 X^{2}-1 ; \\
& T_{3}=4 X^{3}-3 X .
\end{aligned}
$$

This polynomial representation disguises the trigonometric form of the Chebyshev functions also defined by:

$$
T_{n}(X)=\cos (n \arccos X) .
$$

Chebyshev polynomials come very close to the minimax principle: the difference between the Chebyshev approximation 
and the initial function is spread evenly over the interval of definition ("equal ripple"). Thorough discussions of these properties are found in many textbooks. The reader can refer to Hamming [8] for a practical user's point of view.

An additional advantage of Chebyshev polynomials comes from their form given in Eq.(7): properly sampled, the weight factor can be removed. To this end, the continuous functions $T_{n}(X)$ are sampled into a set of discrete functions $T_{j}\left[X_{i}\right]$ that are orthogonal over two sets of abscissa equally spaced in angle $\theta$. We use the set $\left\{X_{i}\right\}$ written over $M$ sample points as:

$$
X_{i}=\cos \left(\theta_{i}\right), \quad \theta_{i}=\pi \frac{i-1 / 2}{M}, \quad i=1, \ldots, M .
$$

The unequal spacing of the points in $X_{i}$ compensates for the weight factor. In addition, each Chebyshev polynomial takes the simple trigonometric form:

$$
T_{n}\left(X_{i}\right)=\cos \left(n \theta_{i}\right)
$$

Hence, we need to evaluate, by interpolations and/or decimations, the backscattered amplitude profiles over $M$ resampled locations $\left\{x_{i}\right\}$ :

$$
x_{i}=\frac{1}{2}\left[\left(x_{\max }+x_{\min }\right)+X_{i}\left(x_{\max }-x_{\min }\right)\right],
$$

in which the dimensionless abscissae $X_{i}$ are derived from Eq.(8).

Then, using such a sampling scheme, the Fourier coefficients are calculated directly with the following sums:

$$
a_{j}[y]=\frac{2}{M} \sum_{i=1}^{M} s_{y}\left(X_{i}\right) \cos \left(j \theta_{i}\right)
$$

with which the approximation up to order $(N-1)$ is simply written:

$$
\sigma_{y}(x)=\frac{a_{0}[y]}{2}+\sum_{j=1}^{N-1} a_{j}[y] T_{j}(X)
$$

where $x$ and $X$ are related by the continuous counterpart of Eq.(10).

\section{$\underline{\text { PROCESSING }}$}

As mentioned in Section $\Pi$, we compute two sets of Fourier coefficients. The first is a complete mapping $\left\{a_{j}[y]\right\}$ of $N=7$ values per ping (and per side). The second set $\left\{a_{j}^{\prime}\right\}$ of $N^{\prime}$ elements is calculated over the single cumulated function:

$$
f(x)=\frac{1}{Y} \sum_{y} s_{y}(x),
$$

$Y$ denoting the total number of pings. The first $N$ coefficients could be derived by simply averaging along-track the previous set $\left\{a_{j}[y]\right\}$ :

$$
a_{j}^{\prime}=\frac{1}{Y} \sum_{y} a_{j}[y], \quad 0 \leq j<N .
$$

However, the order needed to approximate and correct $f(x)$ is much higher $\left(N^{\prime}=30\right)$ than for the first set, so that Eq.(11) must be used for the calculation.

Both sets are then filtered to give respectively the new sets $\left\{\bar{a}_{j}[y]\right\}$ and $\left\{\bar{a}_{j}{ }_{j}\right\}$. For the sake of clarity, let us preview the final steps of the process before going into the details of the filter implementation.

The initial coefficients $a_{j}[y]$ and $a^{\prime}{ }_{j}$ are subtracted from the filtered coefficients $\bar{a}_{j}[y]$ and $\bar{a}_{j}^{\prime}$ to calculate the new set $\left\{a^{\prime \prime}{ }_{j}\right\}$ :

$$
\begin{array}{ll}
a^{\prime \prime}{ }_{j}[y]=\bar{a}_{j}[y]-a_{j}[y]+\bar{a}_{j}{ }_{j}-a_{j}{ }_{j}, & 0 \leq j<N, \\
a^{\prime \prime}{ }_{j}=\bar{a}_{j}{ }_{j}-a_{j}^{\prime}, & N \leq j<N^{\prime},
\end{array}
$$

which, following Eq.(12), yields the additive mapping of the correction to be applied:

$$
c_{y}(x)=\frac{a^{\prime \prime}{ }_{0}[y]}{2}+\sum_{j=1}^{N-1} a^{\prime \prime}{ }_{j}[y] T_{j}(X)+\sum_{j=N}^{N^{\prime}-1} a^{\prime \prime}{ }_{j} T_{j}(X) .
$$

Adding this mapping to the original image yields the corrected image

$$
\bar{s}_{y}(x)=s_{y}(x)+c_{y}(x) .
$$

Implementation of the filters must be done very carefully in order to avoid removing "true" seafloor features. For cumulated pings, the filtered set $\left\{\bar{a}_{j}{ }_{j}\right\}$ is derived from $\left\{a_{j}{ }_{j}\right\}$ through a simple taper with windows $A[j]$ :

$$
\begin{array}{ll}
\bar{a}_{0}^{\prime}=a_{0}^{\prime}, \quad(A[0]=1) \\
\bar{a}_{j}^{\prime}=A[j] a_{j}^{\prime}, \quad 1 \leq j<N^{\prime} .
\end{array}
$$

The coefficient of order $\theta$ is not changed to avoid an alteration of the absolute levels, so that $\bar{a}_{0}^{\prime}-a_{0}^{\prime}$ in Eq.(15) is always zero. Any non-zero value would shift the grey level histogram of the image. The choice of the parameter $N^{\prime}$ is related to the shape of the window $A[j]$. However, we found that the precise shape of the window is not very critical. We chose a parabolic taper:

$$
A[j]=\left[\frac{j-1}{N^{\prime}-1}\right]^{2}, \quad 1 \leq j<N^{\prime},
$$

so that the first order component $\boldsymbol{a}^{\prime}{ }_{1}$, i.e. the average grey level slope, is completely removed from the image $\left(\bar{a}_{1}^{\prime}=A[1]=0\right)$, whereas the higher average components are decreasingly weakened, up to order $N^{\prime}$ which is not altered at all. In selecting $N^{\prime}$, one must keep in mind that the higher the degree of the corresponding polynomial, the smaller the size of the across-track features that are likely to be altered, or even introduced when things go wrong. In our algorithm, $N^{\prime}$ is an adjustable parameter, but several tests showed that a value around 30 usually gives satisfactory results with the parabolic window.

The y-filtering of the set $\left\{a_{j}[y]\right\}$ gives rise to much more severe problems. First, this process must cope with occasional missing data. These bad pings introduce outliers in the $\{a[y]\}$ series. The easiest and cheapest way to overcome this problem is to pre-process the series $\left\{a_{j}[y]\right\}$ with a 3 -points median filter. Then, a low-pass FIR filter must be applied as we want to smooth the local variations occurring between adjacent pings, for each component $a_{j}$. This is achieved by convolving each series with a window $b_{j}[y]$ whose area is unity:

$$
\bar{a}_{j}[y]=a_{j}[y]^{*} b_{j}[y], \quad 0 \leq j<N .
$$

Because we are dealing with a series of coefficients, the shape of the windows was found to be less critical than its size, so we again chose parabolic tapers, over an odd number of points to be able to center the window. Thus, a typical window of width $p=2 q+1$ is written:

$$
b[y]=\frac{6}{p(p+1)(p+2)}\left[(q+1)^{2}-y^{2}\right], \quad-q \leq y \leq q .
$$

There must be a relation $p[j]$ between the size $p$ of the window and the order $j$ of the filtered component. As before, the pertinent property to be remembered for properly setting this part of the processing is that the spatial wavelength of each mode is directly referenced to the swath width.

The $0^{\text {th }}$ order is treated separately from the others, and our program allows a specific length $p[0]$ to be set. By using a large value, e.g. typically more than 100 pings, it enables to correct manual gain changes made during the data recording, even if one does not know the schedule of these changes, or if their timing is uncertain. A more drastic method to obtain the same average grey 
level $a_{0}$ for every ping of the frame is to set in Eq.(15):

$$
a^{\prime \prime}{ }_{0}[y]=a_{0}-a_{j}[y] \text {. }
$$

Side effects of this extreme solution are loss of information about the actual levels of seafloor acoustic backscatter recorded, and a potential increase in amplitude clipping because the initial histogram is shifted.

The size $p$ of the windows $b_{j}[y]$ must be decreasing with the order $j$ of the filtered coefficients. For a given window, the same amount of along-track smoothing is less likely to remove slowly changing geomorphologic features than other patterns evolving rapidly along a short range of pings. As the minimum effective (odd) width of a window is $p=3$, we have linked the decreasing law $p[j]$ to the maximum number $N$ of processed components:

$$
p[j]=2[(N-1) \% j]+1, \quad 1 \leq j<N .
$$

(the symbol $\%$ stands for the arithmetic division)

Therefore, the window width is inversely proportional to $\mathrm{j}$ in the same way the spatial wavelength is inversely proportional to the component's order. As a result, the pertinent parameter is the number of filtered orders $N$, which we set to a small fraction of the ratio, $R$, between the width of the image and the along-track ping spacing:

$$
N=R \% 20+1 \text {. }
$$

The reduction factor (20) between $N$ and the ratio $R$ is meant to preserve high order components. In other words, it allows to process without discrepancies images with an anisotropy of $1 / 20$ between the across-track and along-track directions.

\section{APPLICATION}

In this section, we apply the processing techniques described above to acoustic images obtained with the SeaMARC II bathymetric sidescan sonar system during a survey of Fieberling Guyot $\left(32^{\circ} .5 \mathrm{~N}, 128^{\circ} \mathrm{W}\right)$ [9]. SeaMARC II transmits a $1 \mathrm{~ms}$ pulse of 11 $\mathrm{kHz}$ on port and $12 \mathrm{kHz}$ on starboard, and the seafloor echoes received are displayed as a function of horizontal range to create an image whose width corresponds to a swath about $10 \mathrm{~km}$ wide. Such an image is shown in Figure $2 a$ where the central line corresponds to the survey track going from top to bottom. This image contains several artifacts. The $V$ shaped pattern seen at the upper part of this image is due to interferences from an other sound source. The narrow stripes running along-track on either side of the central gap are due to a sea surface reflection of the near-nadir bottom returns. The consistent along-track nonuniform brightness is particularly obvious on the starboard side. The whole image is also very inhomogeneous when comparing adjacent pings. Finally, data have not been properly recorded in a small area at the top, and also during a few other individual pings along this track.

The approximations $\sigma_{y}(x)$ (Eq.12) derived from the original image $s_{y}(x)$ (Fig. 2a) are displayed in Figure 2b. The order of the approximation has been limited to $N=7$. Although this order is very low, most of the obvious artifacts we want to remove are found in this representation.

The correction coefficients mapping, $c_{y}(x)$ (Eq.16), is displayed in Figure 2c, with a constant grey level offset to bring out both positive and negative corrections. Adding this mapping (Eq.17) to the original image (Fig. 2a) yields the corrected image shown in Figure 2d.

Although the number of filtered orders is limited to $\mathrm{N}=7$ (Eq.24), a noticeable improvement in the image appearance results from this processing. This entails that the defects that we tried to remove actually alter only the very low modes, and that this correction is very likely to remain neutral with respect to the seafloor echo signatures when analyzed by the end users.

From a practical point of view, it must be mentioned that the Chebyshev analysis is not difficult to perform. The computation of the Fourier coefficients with Eq.(11), and the correction mapping with Eq.(16) make use of several samplings of cosine functions. These evaluations must be done only once and then stored for all subsequent uses, i.e. for each ping. The last member of Eq.(16) is also computed only once. A theoretical difficulty may arise from the resampling of the backscatter records, described by Eq.(10). The raw data in the SeaMARC II system consists of 984 samples per side. However, resampling over about 256 points, using rough methods of decimation and interpolation yielded satisfactory results. With a slower computer, even a smaller number of samples $M$ could be used without significant loss of quality: the only requirement is that the highest order $N^{\prime}$ of the decomposition must remain about twice as small as $M$.

\section{CONCLUSION}

We have presented an efficient technique to remove artifacts from a sidescan sonar image of the seafloor that only requires the straightforward computation of a few coefficients per ping. A visual comparison of Figures $2 \mathrm{a}$ and $2 \mathrm{~d}$ underscores the benefits and limitations of the method. Strongly contrasted narrow artifacts are still apparent in Figure 2d, and require further specific processing (e.g. surface multiple, external sound source interferences). For the method to work satisfactorily, the original image must not contain significant gaps, i.e. either missing data or saturated records.

This technique does not require deterministic parameters such as the time varying gain applied by the system or the beam pattern of the sonar. It is purely stochastic and has the advantage of retaining the absolute amplitude levels of the echoes displayed. Nevertheless without additional image enhancements, it brings out features in the final image, that were difficult to see in the original image (Fig 2a lower left). If the absolute values are not needed, the processing can also be set to correct for variations due to manual gain changes imparted at the time data were recorded without requiring a priori knowledge of these changes. Finally, although no information has been added, the visual inconvenience introduced by a few very bad pings can be reduced as well.

\section{ACKNOWLEDGEMENTS}

We wish to thank C. Nishimura and A.N. Shor for help with SeaMARC II data formats and discussions of their processing techniques. We gratefully acknowledge support for this work by the Office of Naval Research through grants N00014-91-J-1073 and N00014-90-J-4009 under management by the Marine Physical Laboratory and the Hawaii Institute of Geophysics respectively. 
a

b

c

d

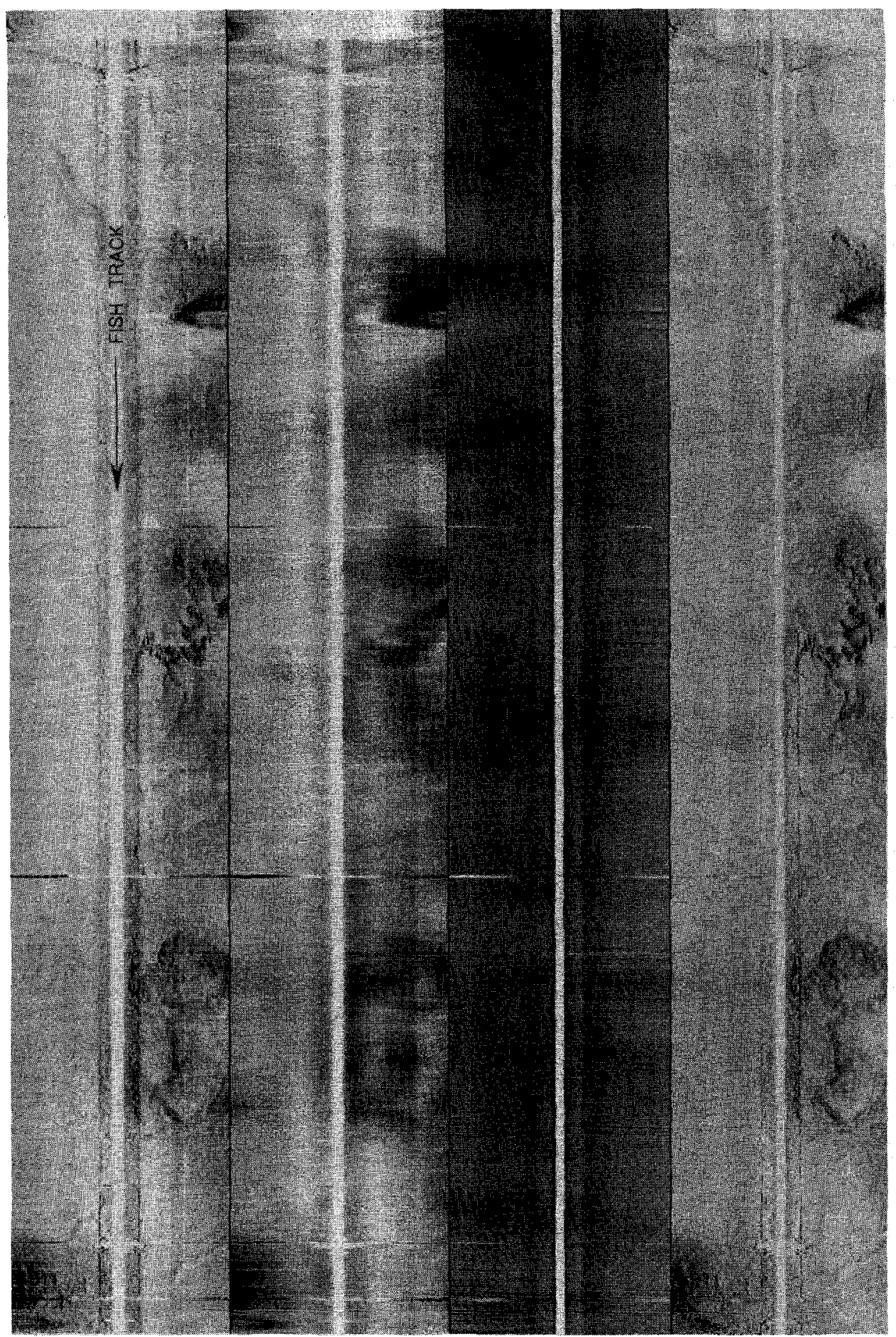

Figure 2 - Processing of SeaMARC II sidescan images. (a) original image of two-sided sidescan data with a $10 \mathrm{~km}$ swath width, (b) approximated image, (c) correction derived from (b) and (d) corrected image. These images have been quantized to 8 bits and printed on Cannon High resolution laser copier. 


\section{REFERENCES}

[1] C.D. Lowenstein, K.A. Kastens and F.N. Spiess, "Display processing for sidescan sonar images", Proc. Offshore Tech. Conf., 1, pp. 49-53, 1980.

[2] B.W. Flemming, "Causes and effects of sonograph distortion and some graphical methods for their manual correction", in Recent Development in Side Scan Sonar Techniques, Russell-Cargill Ed. pp. 103-138, 1982.

[3] P.S. Chavez, "Processing techniques for digital sonar images from Gloria", Photogrammetric Eng. and Remote Sensing., 52, pp. 1133-1145, 1986.

[4] J.M. Augustin, "Side scan acoustic images processing software", Proc. Working Symp. on Oceanographic Data Systems, San Diego CA, pp. 221-228, 1986.

[5] T.B. Reed and D.M. Hussong, "Digital image processing techniques for enhancement and classification of SeaMARC II sidescan sonar imagery", J. Geophys. Res. 94, pp. 7469$7490,1989$.

[6] A. Malinverno, M.H. Edwards, W.B.F. Ryan, 'Processing of SeaMARC swath sonar data", IEEE J. Oceanic Eng., 15(1), pp. 14-23, 1990.

[7] D.T. Cobra, "Estimation and correction of geometric distortions in side-scan sonar images", PhD thesis, MIT 1990.

[8] R.W. Hamming, "Numerical methods for scientists and engineers," chap. 25 to 29, Dover publications, NY, 1973.

[9] C. de Moustier, P.F. Lonsdale and A.N. Shor, "Simultaneous operation of the Sea Beam multibeam echo-sounder and the SeaMARC II bathymetric sidescan sonar system," IEEE J. Oceanic Eng., 15(2), pp. 84-94, 1990. 